\title{
LA-UR-20-29592
}

Approved for public release; distribution is unlimited.

Title: Deterministic Neutron Transport at LANL: The PARTISN Code

Author(s): $\quad$ Davis, Erin Jessica

Intended for: $\quad$ Seminar at UNM

Issued: $\quad 2020-11-19$ 
Disclaimer:

Los Alamos National Laboratory, an affirmative action/equal opportunity employer, is operated by Triad National Security, LLC for the National Nuclear Security Administration of U.S. Department of Energy under contract 89233218CNA000001. By approving this article, the publisher recognizes that the U.S. Government retains nonexclusive, royalty-free license to publish or reproduce the published form of this contribution, or to allow others to do so, for U.S. Government purposes. Los Alamos National Laboratory requests that the publisher identify this article as work performed under the auspices of the U.S. Department of Energy. Los Alamos National Laboratory strongly supports academic freedom and a researcher's right to publish; as an institution, however, the Laboratory does not endorse the viewpoint of a publication or guarantee its technical correctness. 


\section{Deterministic Neutron Transport at LANL: The PARTISN Code}

Seminar for UNM

Erin J. Davis, CCS-2

November 24, 2020

- Los Alamos

NATIONAL LABORATORY 


\section{Neutron Transport}

- The Linearized Boltzmann transport equation describes the bulk motion of neutrons in a material

$$
\begin{gathered}
\left(\frac{1}{v} \frac{\partial}{\partial t}+\widehat{\Omega} \cdot \vec{\nabla}_{r}+\Sigma_{t}\right) \psi(\vec{r}, \widehat{\Omega}, E, t)=\dot{Q}(\vec{r}, \widehat{\Omega}, E, t)+ \\
\int_{4 \pi} d \widehat{\Omega}^{\prime} \int_{E_{\min }}^{E_{\max }} d E^{\prime} \Sigma_{s}\left(\widehat{\Omega}^{\prime} \cdot \widehat{\Omega}, E^{\prime} \rightarrow E\right) \psi\left(\vec{r}, \widehat{\Omega}^{\prime}, E^{\prime}, t\right)+\int_{E_{\min }}^{E_{\max }} d E^{\prime} \bar{v}_{f}\left(E^{\prime} \rightarrow E\right) \phi\left(\vec{r}, E^{\prime}, t\right) \\
\text { Bnitial condition: } \psi\left(\vec{r}, \widehat{\Omega}, E, t_{0}\right)=0 \\
\text { Boundary condition: } \psi\left(\vec{r}_{B}, \widehat{\Omega}, E, t\right)=0, \widehat{\Omega} \cdot \overrightarrow{\mathrm{n}}_{B}>0
\end{gathered}
$$

- This equation is a time-dependent integro-differential equation with seven degrees of freedom for 3-D (six for 2-D, four for 1-D sphere/slab)

- Describes the mean neutron behavior (we'll get back to this!)

- All of the information about how the neutrons interact with the material is incorporated via the cross sections, $\Sigma_{x}$ 


\section{Neutron Diffusion}

- Integrating over angle gives:

$$
\begin{aligned}
\left(\frac{1}{v} \frac{\partial}{\partial t}+\Sigma_{t}\right) \phi(\vec{r}, E, t) & +\vec{\nabla}_{r} \cdot \int_{4 \pi} d \Omega \widehat{\Omega} \psi(\vec{r}, \widehat{\Omega}, E, t)=\int_{4 \pi} d \Omega \dot{Q}(\vec{r}, \widehat{\Omega}, E, t)+ \\
\int_{E_{\text {min }}}^{E_{\max }} d E^{\prime} \Sigma_{s}\left(E^{\prime} \rightarrow E\right) \phi\left(\vec{r}, E^{\prime}, t\right) & +\int_{E_{\min }}^{E_{\max }} d E^{\prime} \bar{v} \Sigma_{f}\left(E^{\prime} \rightarrow E\right) \phi\left(\vec{r}, E^{\prime}, t\right)
\end{aligned}
$$

- Apply Fick's Law: $\vec{J}=\int_{4 \pi} d \Omega \widehat{\Omega} \psi(\vec{r}, \widehat{\Omega}, E, t) \approx-D(\vec{r}, E) \vec{\nabla}_{r} \phi(\vec{r}, E, t)$

$$
\begin{aligned}
\left(\frac{1}{v} \frac{\partial}{\partial t}+\Sigma_{t}\right) \phi(\vec{r}, E, t)-\vec{\nabla}_{r} \cdot D(\vec{r}, E) \vec{\nabla}_{r} \phi(\vec{r}, E, t)=\dot{\mathcal{Q}}(\vec{r}, E, t)+ \\
\int_{E_{\min }}^{E_{\max }} d E^{\prime} \Sigma_{s}\left(E^{\prime} \rightarrow E\right) \phi\left(\vec{r}, E^{\prime}, t\right)+\int_{E_{\min }}^{E_{\max }} d E^{\prime} \bar{v} \Sigma_{f}\left(E^{\prime} \rightarrow E\right) \phi\left(\vec{r}, E^{\prime}, t\right)
\end{aligned}
$$

- Smaller phase space, but only accurate for isotropic/weakly-anistropic fluxes 


\section{Neutron Transport: Solution Methods}

\section{Monte Carlo (Stochastic)}

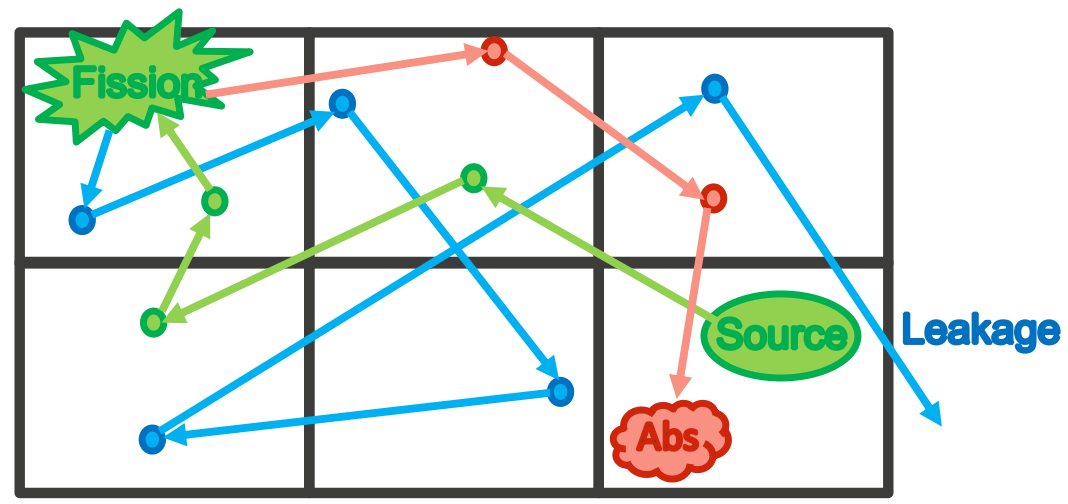

- Models the 'random walk' taken by particles through the system-the energy, angle and location of a particle are discrete quantities

- Discrete events are sampled from probability density functions (cross sections)

- If we are interested in the mean, then we must run enough histories to get good statistics (reasonably smooth distribution function)
SN/Discrete Ordinates (Deterministic)

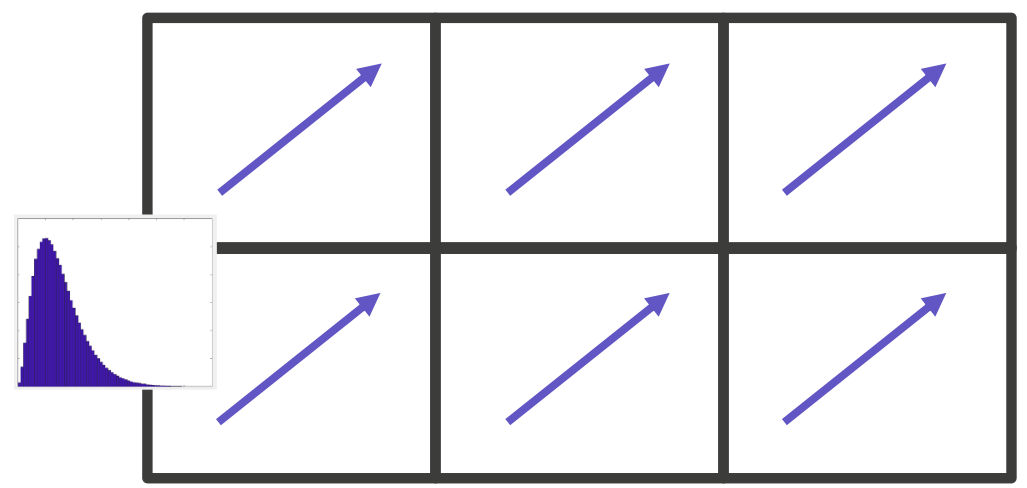

- Numerically solve a PDE

- Trace the distribution function through space along discrete velocities (angle + energy)

- Derivatives in time and space move particles between time steps and spatial cells

- Changes in energy and direction due to scattering and fission (absorption and reemission) events lead to coupling in the equations for the energy groups and angles

- Derivatives in direction for curvilinear geometries 


\section{Neutron Transport: Solution Methods}

\section{Monte Carlo (Stochastic)}

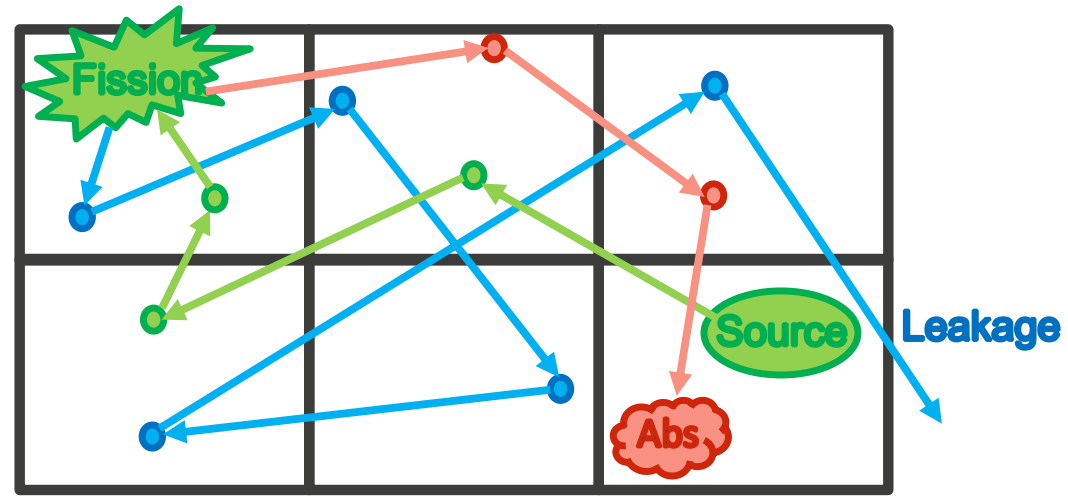

1. To improve accuracy, must reduce the variance $\rightarrow$ More particle histories

2. Variance reduction via source biasing, weight windows, Russian Roulette etc.

3. Can struggle with obtaining a smooth solution due to noise

4. Can use continuous energy or multigroup cross sections
SN/Discrete Ordinates (Deterministic)

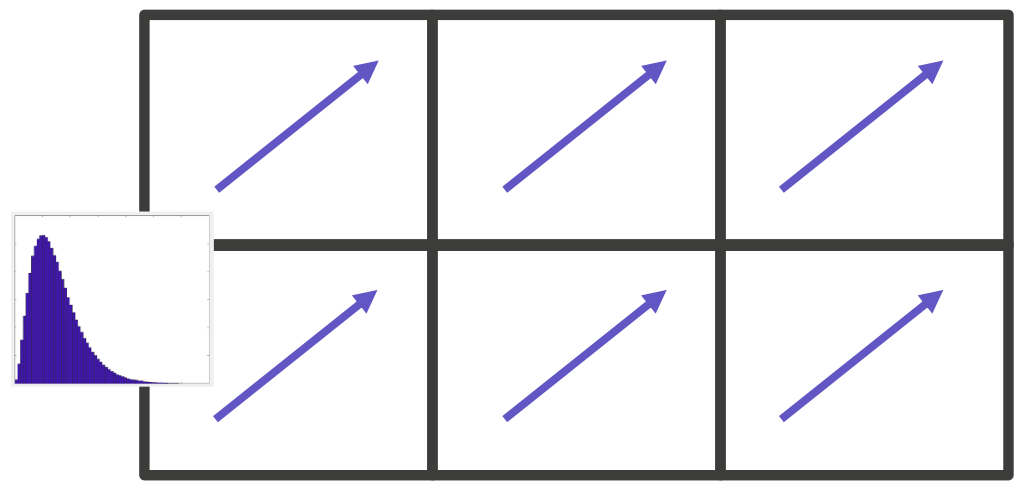

1. To improve accuracy, add unknowns $\rightarrow$ more angles and groups (sometimes), smaller spatial cells and time steps

2. This does not have to be done uniformlyrefine in 'important' regions of phase space

3. Can obtain a smooth solution, but subject to discretization errors, ray effects, negative fluxes

4. Must use multigroup cross sections 


\section{Neutron Cross Sections}

- Evaluated through a combination of experimental results, theoretical models and expert judgment

- Resonances due to discrete excitation energies of atomic nuclei

- 'Continuous energy' cross sections may contain upwards of $10^{4}$ points!

- The greatest source of uncertainty in almost any transport calculation

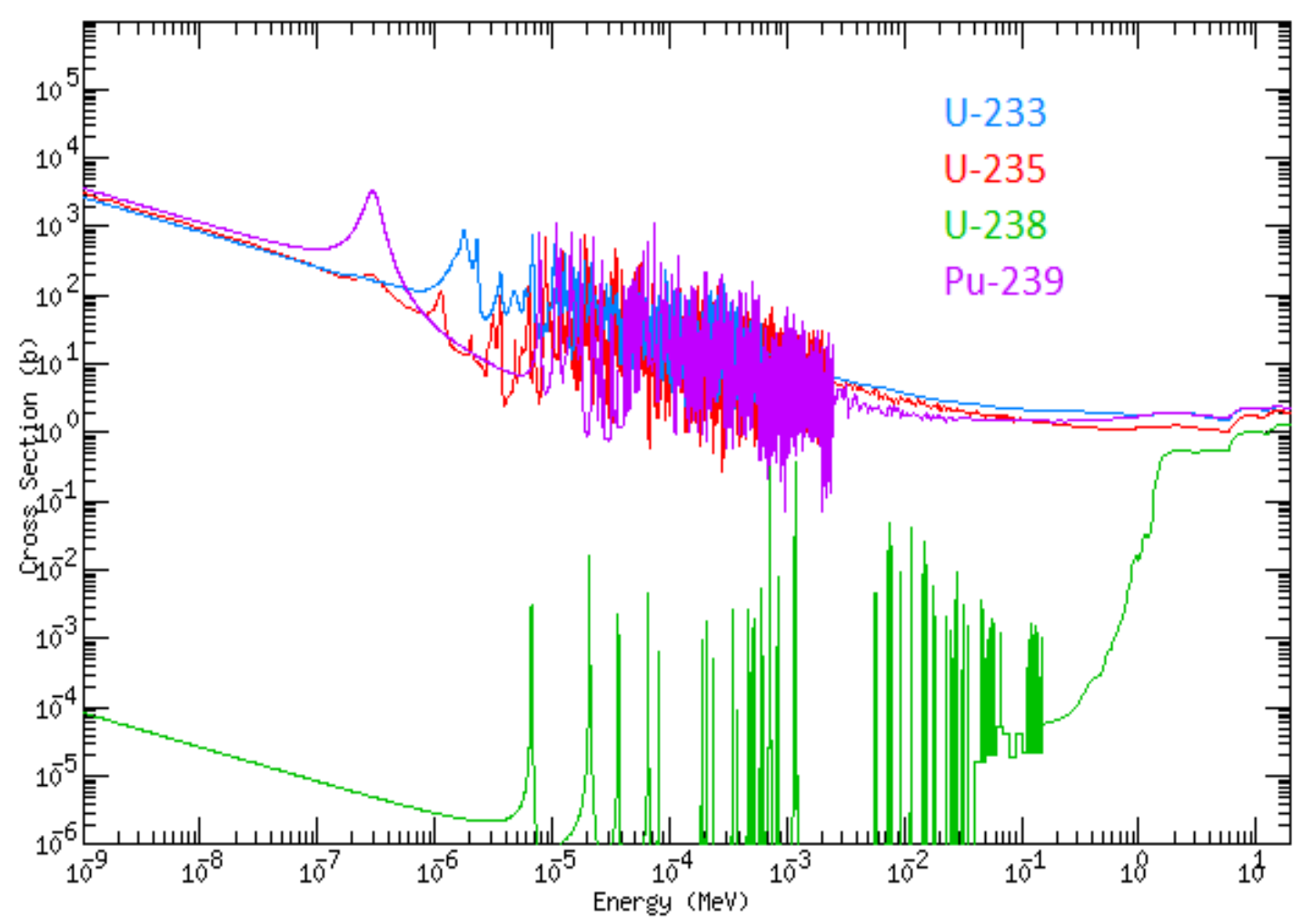

Figure: Fission Cross Sections of Various Isotopes 


\section{Neutron Cross Sections-Multigroup-in-Energy}

- Monte Carlo codes can use continuous energy cross sections

- Deterministic codes must discretize in energy $\rightarrow$ Multigroup

- Assume that the energy spectrum of the neutrons is known $\rightarrow$ 'weight function'

- Choose energy group boundaries carefully

- Use the weight function and energy group boundaries to generate 'multigroup cross sections'

- Adjust the multigroup cross sections by tuning them to match experimental criticality benchmarks

- Multigroup cross section sets are tailored to specific applications

- Can also be used in for Monte Carlo $\rightarrow$ More efficient sampling of the cross section

- Resonance self-shielding can be an issue (we'll come back to this)

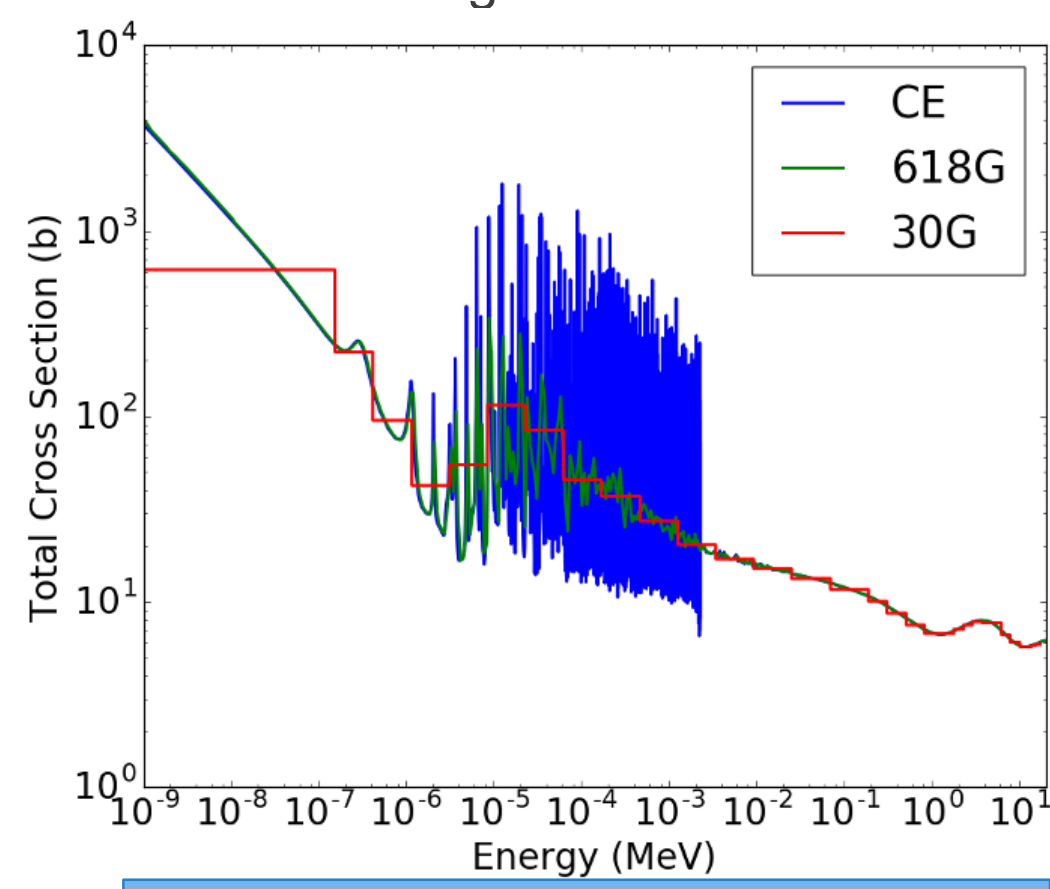

Figure: U-235 Total Cross Section (Thomas Saller) 


\section{Angles + Energy = Velocity!}

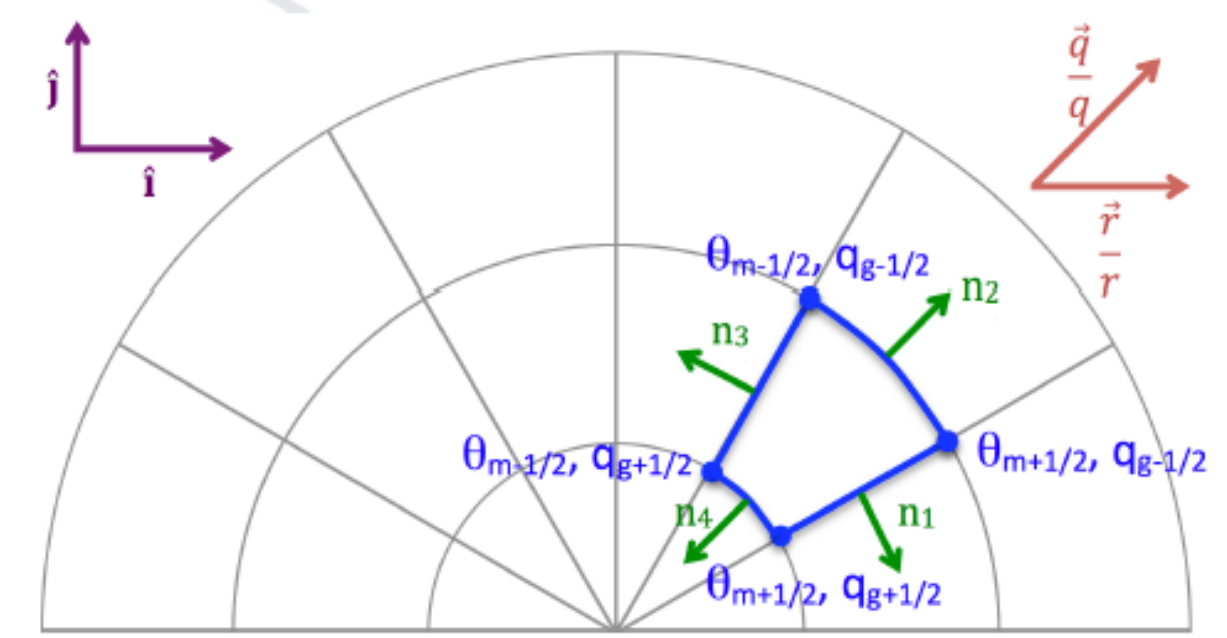

Velocity Mesh
- Solving the transport equation involves taking integrals over angle

- The angular variable, $\widehat{\Omega}$, is discretized using numerical quadrature, which also provides a way to do those integrals:

$$
\int_{4 \pi} d \widehat{\Omega} \psi(\widehat{\Omega}) \approx \sum_{n=1}^{N} w_{n} \psi\left(\widehat{\Omega}_{n}\right)
$$

- The transport equation is solved for discrete directions of travel, $\widehat{\Omega}_{n}$ 


\section{PARTISN Code Capabilities}

- PARTISN: PARallel, TIme-dependent SN

- Solves both the static and time-dependent forms of the linear Boltzmann transport equation

- Forward and adjoint mode

- Static Eigenvalues: $k$ and $\alpha$

- Time-dependent stochastic neutron transport equations:

- Probability of Initiation (static) \& Probability of Survival: Probability of obtaining a divergent chain

- Moments of the (instantaneous) neutron and (total) fission numbers

- Discretization strategies:

- Discrete ordinates approximation for treating the angular variable

- Multigroup in energy

- Diamond difference (standard or adaptive weighted) for the spatial variable

- Crank-Nicholson in time

- Set-to-zero fixup to maintain positivity (negative sources, strong gradients, large spatial cells)

- Acceleration via diffusion synthetic acceleration method or the transport synthetic acceleration

- Parallelization is performed via 2-D spatial decomposition ("KBA") and/or Jacobi iteration for energy decomposition 


\section{Radiation Transport Applications in CCS-2}

The transport project in CCS-2 develops neutron and $\mathrm{x}$-ray transport codes

- Neutral particle, linear transport - Nuclear reactor simulations

- Criticality (k-effective)

- Isotope depletion

- Shielding

- Medical, e.g., brachytherapy

- Oil well logging

- Supernova explosion

- Nonlinear transport of thermal x-rays - Inertial confinement fusion

- National Ignition Facility (Lawrence Livermore)

- Laboratory for Laser Energetics (Rochester)

- Astrophysics (Center for Theoretical Astrophysics, Los Alamos)

- Z Pulsed-Power machine (Sandia)

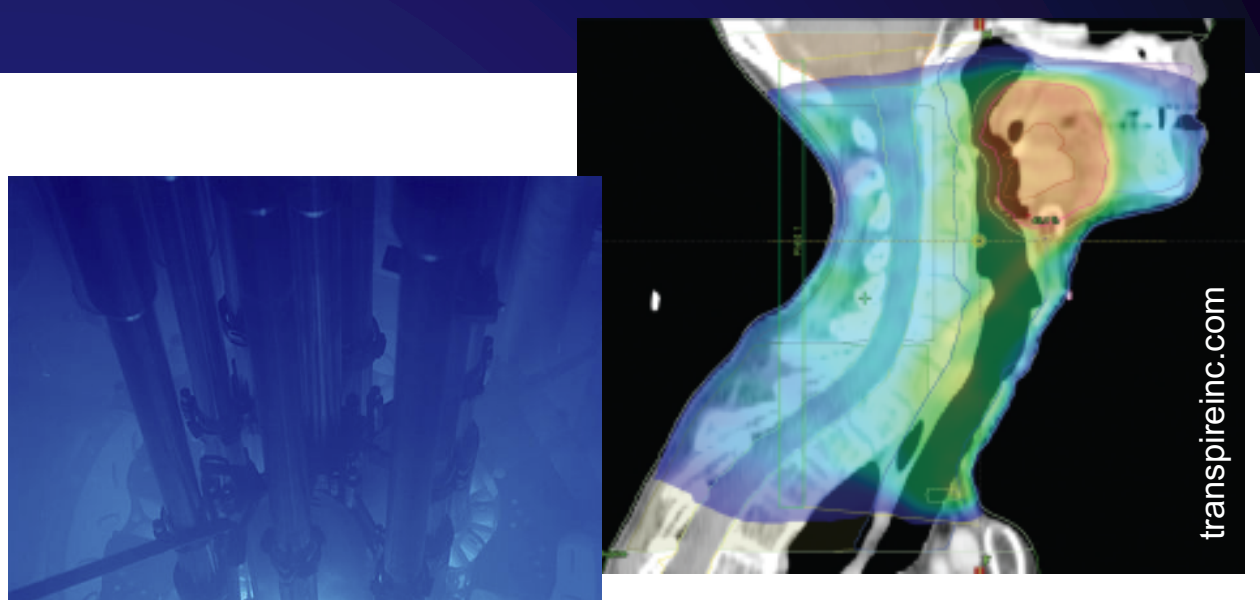




\section{PARTISN - Neutron/Gamma Deterministic Transport}

Our team adds new physics, develops better solution methods, translates these into software and helps our users understand their results

- Our worldwide users perform a wide variety of 1-D/2-D/3-D calculations, so we require robust and efficient methods for reducing transport solution iterations (e.g., "KBA" sweeps, Diffusion Synthetic Acceleration)

- We have long been involved with work and research in nuclear application areas such as criticality safety, reactor engineering/shielding, sensitivity analysis, inverse problems, etc.

- We are also applying our methods and solvers to non-traditional areas such as the r-process in core-collapse in supernovae and neutronstar mergers $\rightarrow$ material-motion effects
Figure: R-Process Nucleosynthesis Rapid neutron capture in these types of events is believed to account for the creation of roughly half the abundance of materials heavier than $\mathrm{Fe}$

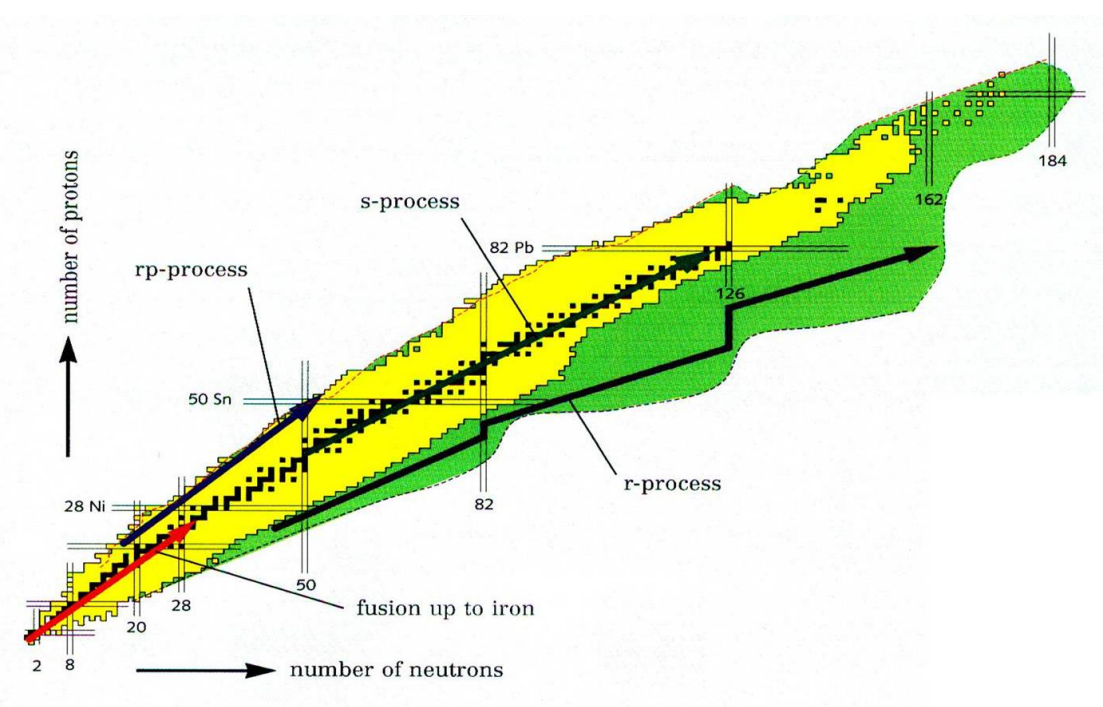




\section{PARTISN - Neutron/Gamma Deterministic Transport}

- Six dedicated team members (5 staff, 1 postdoc) who spend varying amounts of time working on the code and supporting LANL users

- Currently, about 113k lines of (mostly) modern Fortran

- Methods development

- Material-motion advection algorithms

- Using machine learning to improve multigroup data

- Self-shielding corrections for multigroup data

- Solutions to the stochastic neutronics equations

- Use of Block Adaptive Mesh Refinement (AMR)

- Efficient and scalable computational transport solution methods for massively parallel architectures using a "MPI+X" paradigm

- Software maintenance

- Bug fixes

- Code modernization

- User support

- Help our users run the code and understand their results

- We generally have at least one summer student intern

- The code is available through RSICC and it is installed on LANL and LLNL machines 


\section{PARTISN Future Research Interests}

- Solution methods for the transport equation with neutron-neutron scattering

- Time-dependent multigroup weighting functions

- Relativistic moving material corrections

- Collaborations with NEN-2 (Advanced Nuclear Technology) on FVM, Rossialpha, etc.

- Neutron-Diagnosed Sub-critical Experiments (NDSE)

- Pre-initiation experiments (GODIVA-IV)

- Anisotropic fission (once data is available) 


\section{Stochastic Neutronics}




\section{Stochastic Neutron Behavior}

\section{Deterministic Regime}

Strong neutron source

Gaussian-like, approaches a delta-function at the mean as the source rate increases

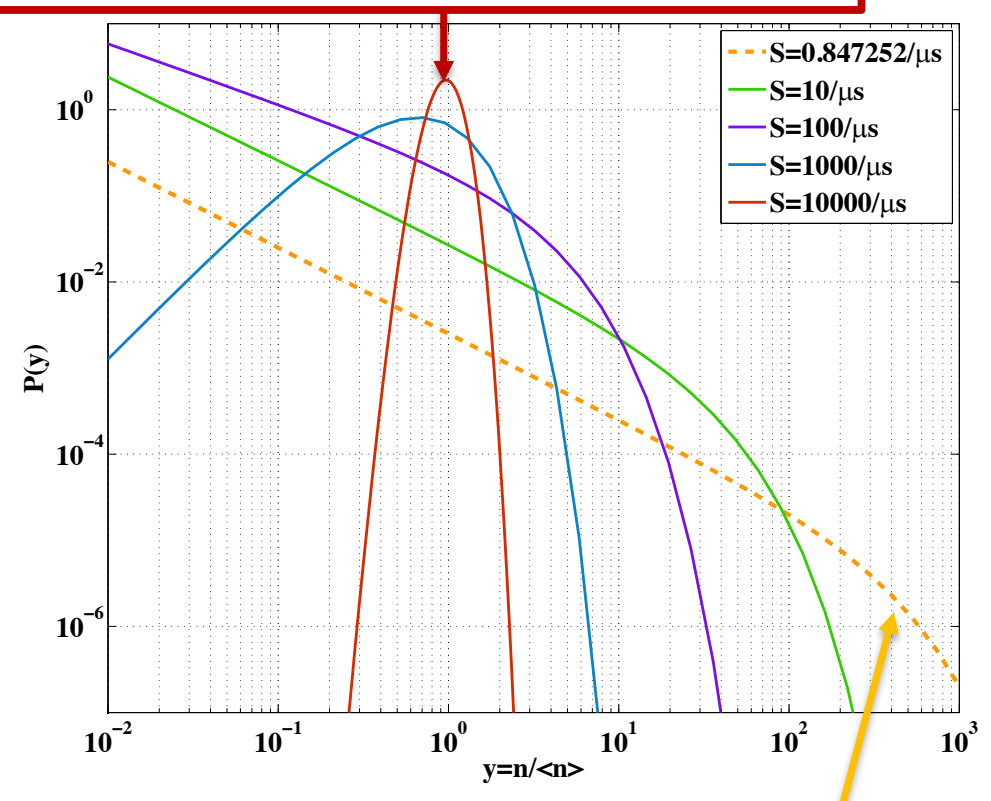

Figure: PDF of $y$ (the ratio of neutron number, $n$, to mean neutron number, $<n>$ ) for various source rates, $S$
Stochastic Regime Weak neutron source, i.e., spontaneous fission Most likely outcome is zero neutrons, but long tail
- Nuclear fission is a fundamentally stochastic process, so the outcome of any one fission is known only probabilistically

- Deterministic behavior of a fissile system is the result of individual fission chains overlapping to the extent that they become indistinguishable

- Mean neutron behavior drives the evolution of the neutron population $\rightarrow$ Predictability!

- Stochastic behavior occurs when the source is weak or the neutron population is very small

- Overlapping does not occur $\rightarrow$ Must account for the inherent stochasticity of fission (number of neutrons emitted is probabilistic)

- This occurs in criticality accident scenarios, passive detection of spontaneous fission sources, reactor startup, criticality excursions in spent fuel, burst reactors, etc. 


\section{Use of "Point-Models" to Describe Stochastic Behavior}

- "Point-Models," for which time is the only phase-space variable, have long been used in neutron transport to generate simple, analytic representations of fissile systems

- Feynman and Bell pioneered their use at LANL for stochastic systems, deriving an analytic gamma distribution that approximates the PDF of $n$, the instantaneous neutron number:

$$
P(n)=\left(\frac{n}{\langle n\rangle}\right)^{\eta-1} \frac{\eta^{\eta}}{\langle n\rangle \Gamma(\eta)} e^{\eta \frac{n}{\langle n\rangle}} \text { for } \eta=\frac{S \cdot P O I}{\alpha}
$$

$-\langle n\rangle$ is the mean neutron number in the system at some point in time, $t_{f}$, given that there were no neutrons at the initial time, $t_{0}$

- Even though the model is based on a point-model, information about the geometry and the neutron velocity can be incorporated by computing $S \cdot P O I$ and $\alpha$ using a full phase-space transport code

- The model is limited by the fact that it does not allow for time-varying geometries

- Bell also derived a system of probabilistic, full phase-space transport equations that can be solved using a deterministic transport code $\rightarrow$ Available in PARTISN 


\section{Use of "Point-Models" to Describe Stochastic Behavior}

- Work by Prinja showed that a related analytic expression could be derived for the cumulative fission number, $f$ :

$$
P(f)=\frac{\sigma_{n}}{\sigma_{f}}\left(\frac{\sigma_{n}}{\sigma_{f}} \frac{f-\langle f\rangle}{\langle n\rangle}+1\right)^{\eta-1} \frac{\eta^{\eta}}{\langle n\rangle \Gamma(\eta)} e^{\frac{\sigma_{n}}{\sigma_{f}} \frac{f-\langle f\rangle}{\langle n\rangle}+1}
$$

$-\langle f\rangle$ is the mean number of fissions that occur on $t \in\left[t_{0}, t_{f}\right]$ and $\sigma_{n}$ and $\sigma_{f}$ are the standard deviations of the distributions

- Number of fissions is directly related to emitted energy and is therefore more relevant than neutron number for quantifying energy release, e.g., analysis of criticality accident scenarios

- Prinja also derived full phase-space equations for the fission number $\rightarrow$ Available in PARTISN 


\section{Full Phase-Space Equations for the Moments of $\boldsymbol{n}$ and $\boldsymbol{f}$}

- The equation for each moment, $\overline{g^{k}}$, is identical in form to the other moments of the fission number, $f$, and the neutron number, $n$

- However, the equations for $\overline{n^{k}}$ and $\overline{f^{k}}$ have different sources and initial conditions

- They equations are standard linear adjoint transport equations with a source term, $S_{k}$, that depends on the lower-order moments $\left(-\frac{1}{v} \frac{\partial}{\partial t}-\widehat{\Omega} \cdot \vec{\nabla}_{r}+\Sigma_{t}\right) \overline{g^{k}}(\vec{r}, \widehat{\Omega}, E, t)=S_{k}\left(\vec{r}, \widehat{\Omega}, E, t ; \bar{g} \cdots \overline{g^{k-1}}\right)+$

$$
\int_{4 \pi} d \widehat{\Omega}^{\prime} \int_{E_{\min }}^{E_{\max }} d E^{\prime} \Sigma_{s}\left(\widehat{\Omega} \cdot \widehat{\Omega}^{\prime}, E \rightarrow E^{\prime}\right) \overline{g^{k}}\left(\vec{r}, \widehat{\Omega}^{\prime}, E^{\prime}, t\right)+
$$

$$
\bar{v} \Sigma_{f}(E) \int_{E_{\min }}^{E_{\max }} d E^{\prime} \chi\left(E \rightarrow E^{\prime}\right) \overline{g^{k}}\left(\vec{r}, E^{\prime}, t\right)
$$

Initial condition: $\overline{n^{k}}\left(\vec{r}, \widehat{\Omega}, E, t_{\text {final }}\right)=1$ \& $\overline{f^{k}}\left(\vec{r}, \widehat{\Omega}, E, t_{\text {final }}\right)=0$

Boundary condition: $\overline{g^{k}}\left(\vec{r}_{B}, \widehat{\Omega}, E, t\right)=0, \widehat{\Omega} \cdot \overrightarrow{\mathrm{n}}_{B}<0$

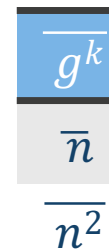

$$
\begin{array}{cc}
\bar{f} & \Lambda_{0} \\
\overline{f^{2}} & \Lambda_{0}+2\langle\bar{f}\rangle\left(\Lambda_{1}+\Lambda_{2}\langle\bar{f}\rangle\right)
\end{array}
$$

$2 \Lambda_{2}\langle\bar{n}\rangle$

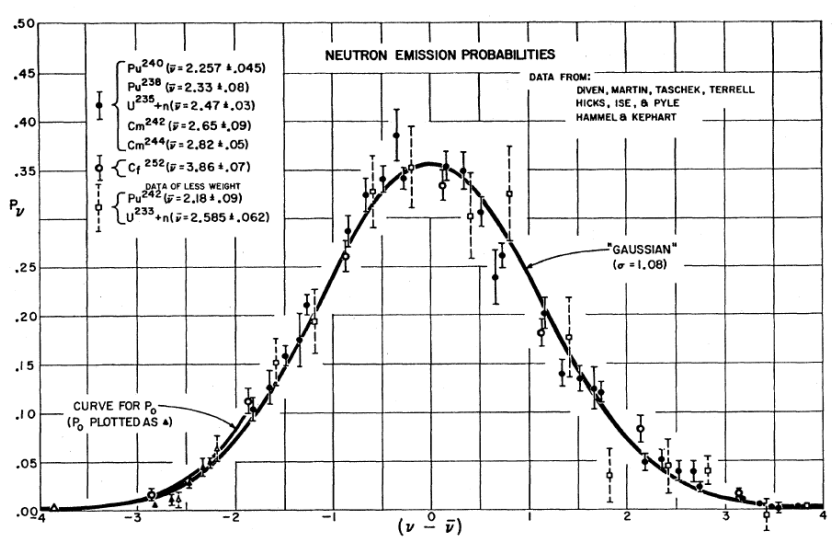

Fic. 4. Experimental noncumulative neutron emission probabilities. The continuous curves are for a "Gaussian"
distribution. Experimental data are from references 14-16; standard deviations are shown.

\section{Table: Source terms}

$$
\Lambda_{k}(E)=\frac{\Sigma_{f}(E)}{i !} \sum_{j=i}^{I} \frac{i !}{(j-i) !} p_{j}
$$$$
\left\langle\overline{g^{k}}\right\rangle=\int_{E_{\min }}^{E_{\max }} d E^{\prime} \chi\left(E \rightarrow E^{\prime}\right) \overline{g^{k}}\left(\vec{r}, E^{\prime}, t\right)
$$

- Accounts for variation in the solution due to the fission multiplicity distribution 


\section{Full Phase-Space Descriptions of Stochastic Behavior}

- Our codes can perform full phase-space computations as well as incorporate changing geometries

- MCATK: Analog Monte Carlo tracks individual fission chains to compute $n$ and $f \rightarrow$ The outcomes are tallied to form the distributions and compute their moments (Travis Trahan)

- PARTISN: Equations for the moments of $n$ and $f$ are solved directly

- The moments can be used to compute the analytic PDF parameter, $\eta$

Table: System parameters computed using MCATK and PARTISN (21 energy groups) for a $6.5 \mathrm{~cm}$ sphere of $\mathrm{Pu}$

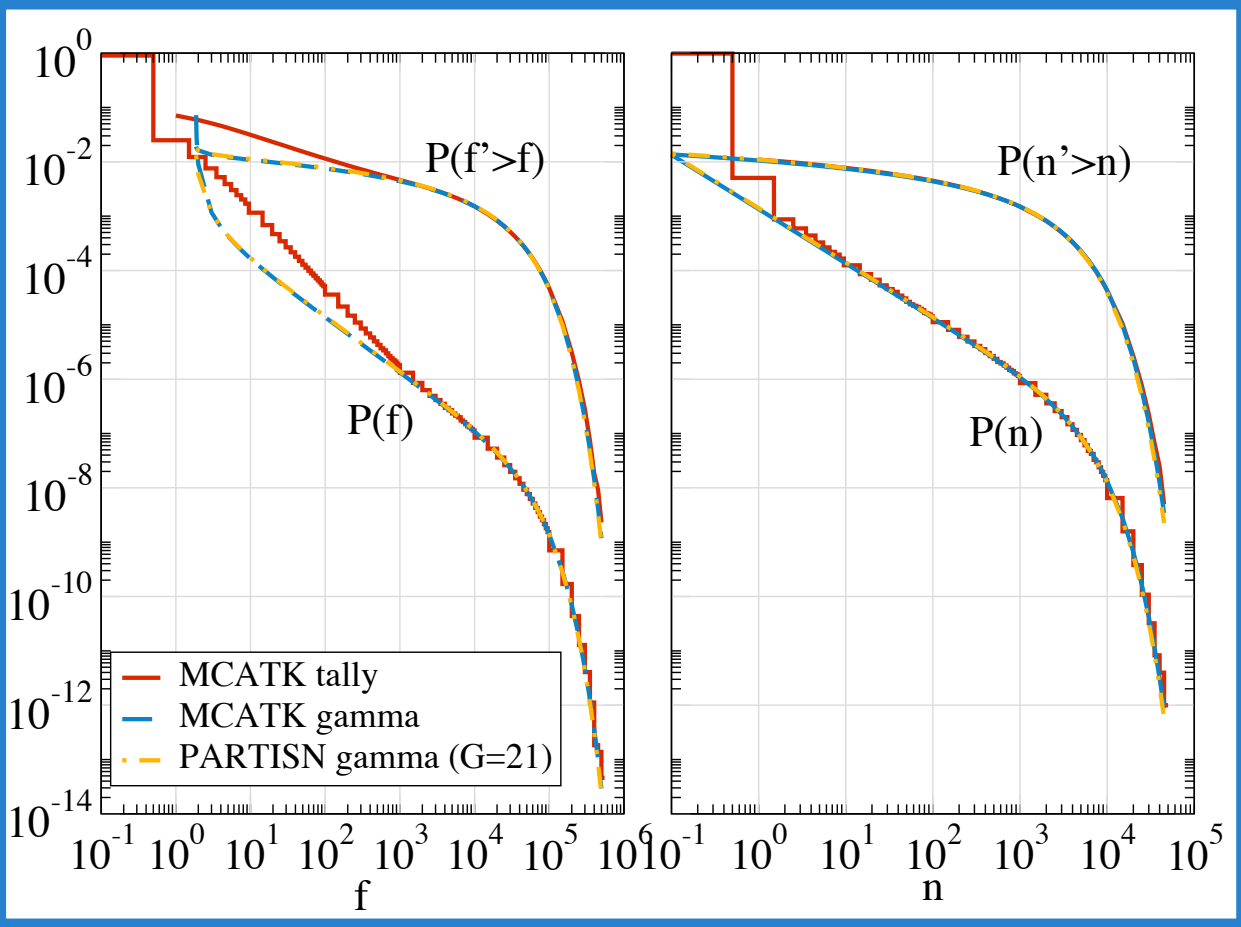

\begin{tabular}{|l|c|c|c|c|c|} 
& $\begin{array}{c}\alpha \\
\left(\mu \boldsymbol{s}^{-1}\right)\end{array}$ & $\eta=\frac{S \cdot P O I}{\alpha}$ & $\eta=\left(\frac{\langle\boldsymbol{n}\rangle}{\sigma_{n}}\right)^{2}$ & $\langle\boldsymbol{n}\rangle$ & $\langle\boldsymbol{f}\rangle$ \\
\hline PARTISN & 7.7184 & $1.5024 \mathrm{e}-3$ & $1.3951 \mathrm{e}-3$ & 5.8661 & 60.625 \\
\hline MCATK & 7.7253 & -- & $1.3641 \mathrm{e}-3$ & 5.8659 & 61.438 \\
\hline
\end{tabular}

Figure: PDFs of $f$ and $n$ at $t_{f}=0.5 \mu s$

1. Direct tally from MCATK (target solution)

2. Analytic gamma PDF with MCATK $\eta$ from table

3. Analytic gamma PDF with PARTISN $\eta$ from table Agreement is good for $n$ and large $f$.

(The discrepancy for small $f$ is due to fission chains that die before $t_{f}$.) 


\section{Full Phase-Space Descriptions of Stochastic Behavior}

- Dynamic Uranium Test Problem

$-17.25 \mathrm{~cm}$ sphere with a time-varying mixture of U-235 and U-238 where $\rho_{235}=14-6 C \frac{\mathrm{g}}{\mathrm{cm}^{3}}$,

$\rho_{238}=1+6 C \mathrm{~g} / \mathrm{cm}^{3}$ and

$C$ varies linearly between time points

- Uniformly distributed volume source in group 7 (14-14.25 MeV) emitting $0.1 \mathrm{n} / \mathrm{s}$

Figure: Moment comparison PARTISN vs. MCATK MCATK results were generated using ENDF-VII.1 data, time biasing and 50 independent runs with 554400 fission chains each (Travis Trahan) PARTISN results generated using MENDF71x data library, 133 groups, 120 spatial cells, S-20 and P-3 scattering/flux moments

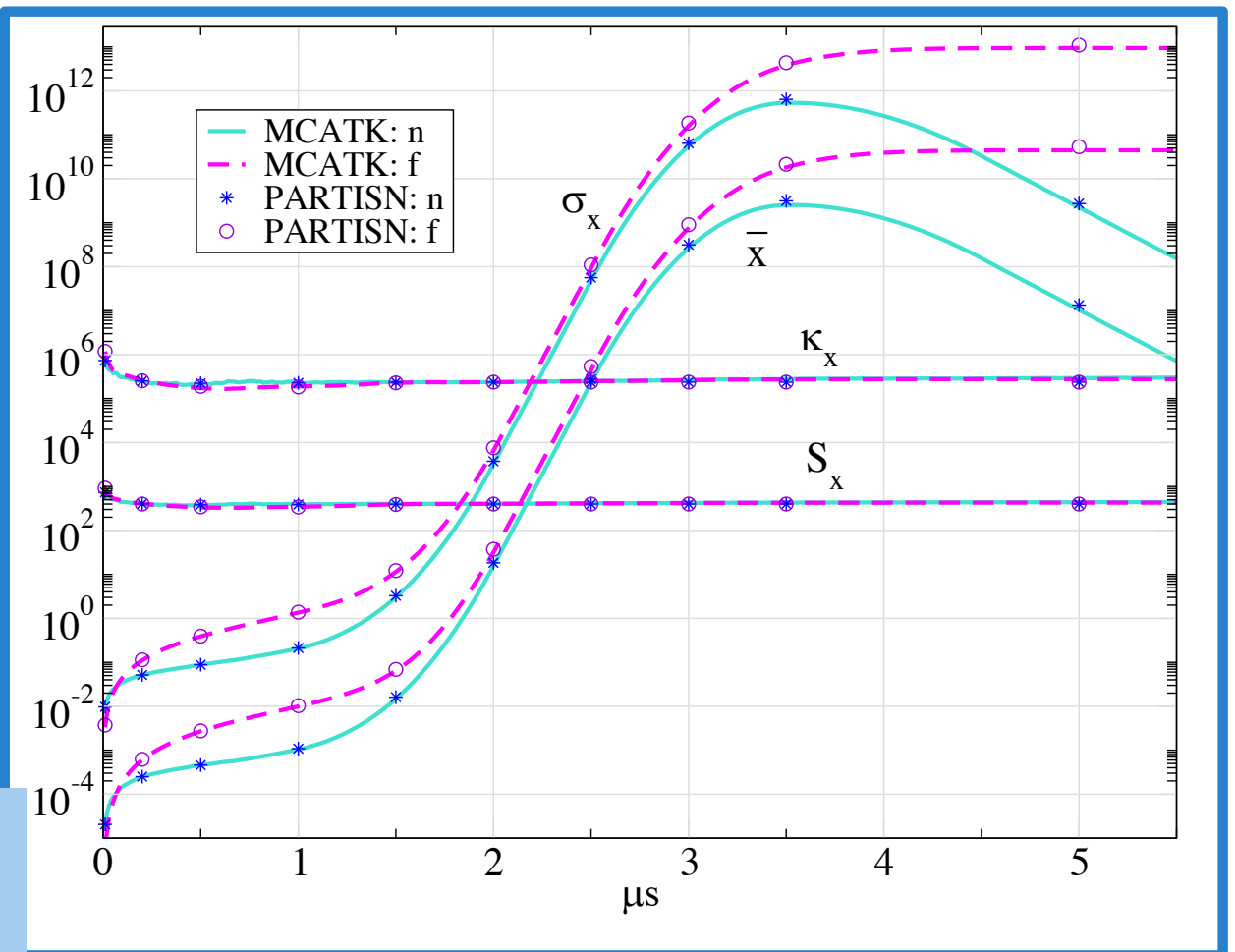




\section{Self-Shielding Corrections Thomas Saller}




\section{The Multigroup Approximation}

- Cross sections are continuous in energy but generally cannot be defined by an analytic function

- In the multigroup approximation, the energy range is broken up into a series of 'groups,' and the cross section for reaction $x$ is averaged over these ranges by some weight function $f(E)$ :

$$
\sigma_{x, g}^{i}=\frac{\int_{E}^{E+\frac{1}{2}} \sigma_{x}^{i-\frac{1}{2}}(E) f(E) d E}{\int_{E_{g+\frac{1}{2}}}^{E g-\frac{1}{2}} f(E) d E}
$$

- Obtaining accurate multigroup cross sections requires an accurate $f(E)$

- The ideal weight function would be the flux of the problem of interest, which is not known a priori $\rightarrow$ use a "best guess"

- At LANL, we use the TD weight function, which consists of a Maxwellian at low energies, a $1 / E$ slowing down range, a fission spectrum at high energies, and a 14.1 MeV fusion peak

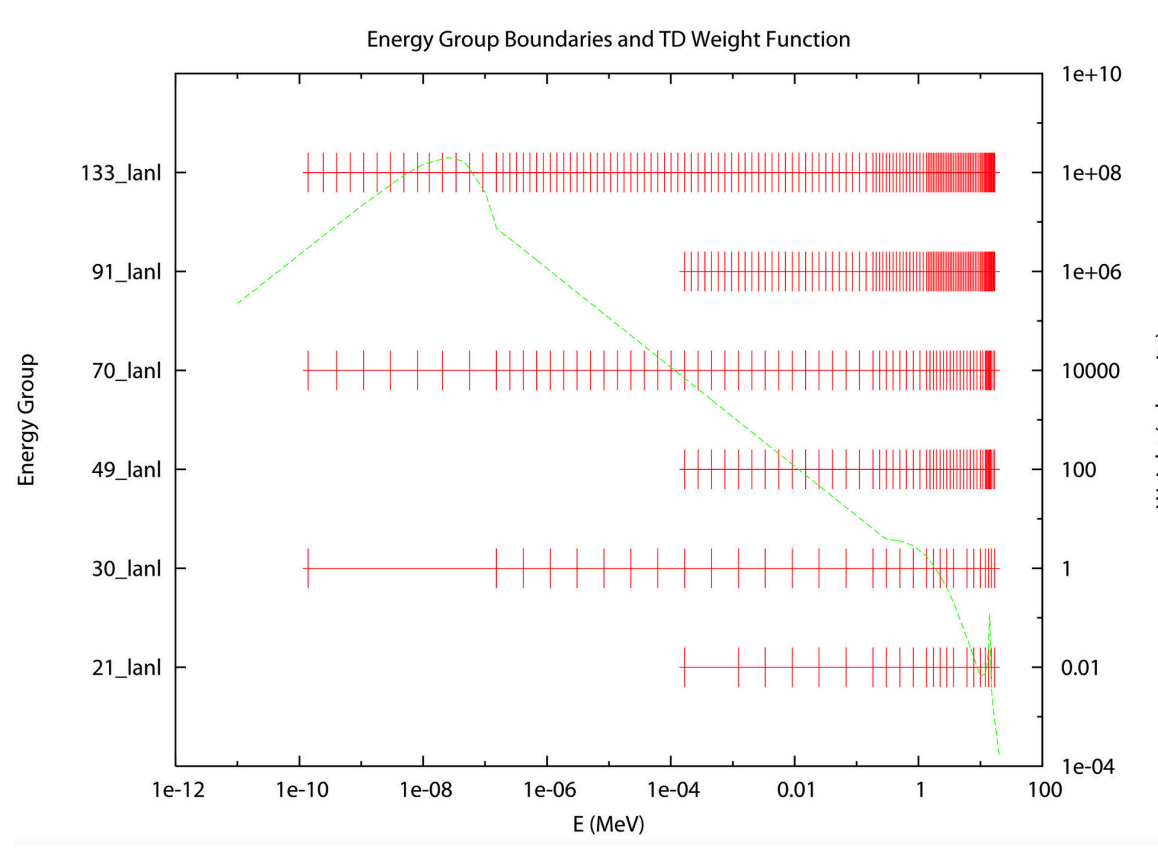

Figure: LANL group structures and weight function 


\section{Self-Shielding Corrections in PARTISN}

- Spectrum changes due to the mixing of isotopes (particularly resonant isotopes) is referred to as "self-shielding"

- PARTISN uses the Bondarenko method to account for self-shielding effects

- In the Bondarenko method, the macroscopic total cross section of a material is broken up into two parts:

1. $\sigma_{t}^{i}(E)$ : Microscopic cross section of isotope $i$

2. $\sigma_{0}^{i}(E)$ : Background cross section, which depends on all of the other isotopes in a material $m$

$$
\Sigma_{t}^{m}(E)=N^{i}\left(\sigma_{t}^{i}(E)+\sigma_{0}^{i}(E)\right)
$$

- To account for spatial effects, we include an "escape cross section," $\Sigma_{e}$, in the background cross section:

$$
\sigma_{0}^{i}=\frac{1}{N^{i}}\left(\Sigma_{e}+\sum_{(j \neq i) \in m} N^{j} \sigma_{t}^{j}\right) .
$$

- The escape cross section relates the outgoing current from a region to the scalar flux:

$$
\Sigma_{e}(E)=\frac{J^{+}(E)}{\phi(E)}
$$




\section{Self-Shielding Corrections in PARTISN}

- The background cross section is used in a cross section processing code (e.g., NJOY) to generate multigroup cross sections as a function of $\sigma_{0}^{i}$

- In general, multigroup cross sections are "infinitely dilute," where $\sigma_{0}=10^{10}$ (no selfshielding correction)

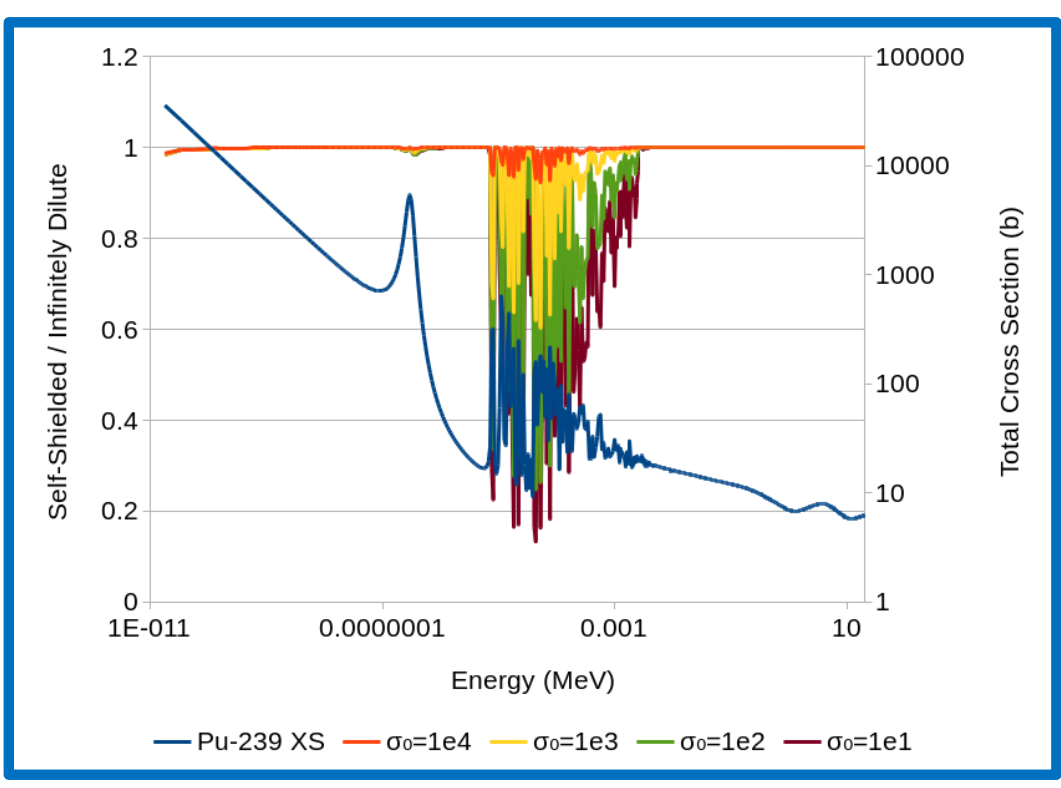

Figure: Pu-239 total cross section as a function of $\sigma_{0}^{i}$
Figure: Uranium sphere with 5 atom-percent U-235 Over time, U-235 is replaced by U-238.

The value of $\alpha$ compares better to MCATK (Monte Carlo) when self-shielding is used. 


\section{Self-Shielding Corrections in PARTISN}

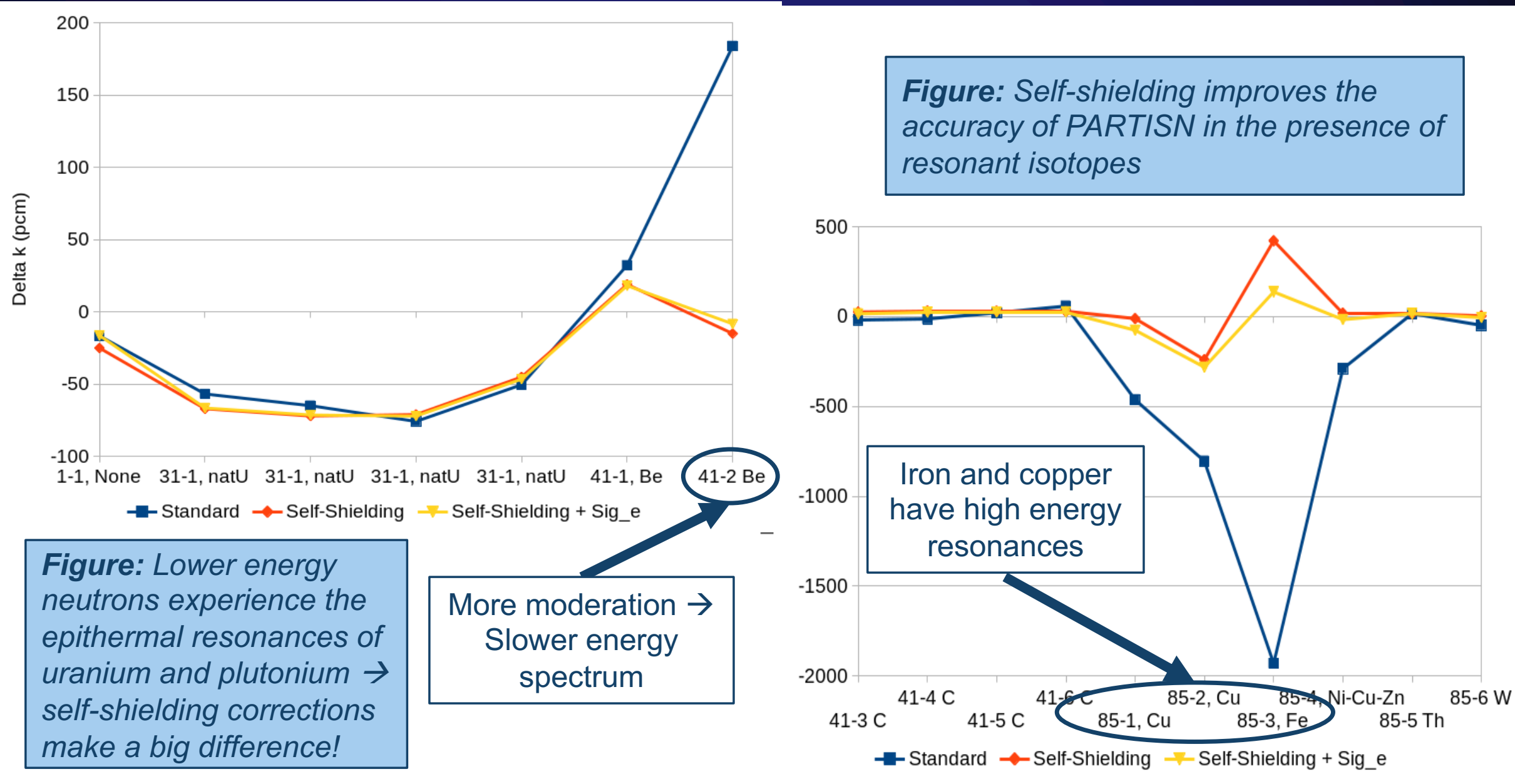




\section{Moving Material Corrections}




\section{Transport in Moving Materials}

- The neutron transport equation is generally applied to systems in which the material is assumed to be at rest

- There are instances where material motion can affect the resulting solution

- Coolant flowing through a nuclear reactor

- Collapsing stellar cores

- When the material is moving, there is a discrepancy between neutron velocities in the comoving \& lab frames $\rightarrow$ neutron cross sections are measured in the lab frame

- Using the wrong cross section results in errors in reaction rates \& the transport solution

- Even though nuclear cross sections are evaluated with respect to a material at rest, it is the relative velocity between the material \& neutrons (i.e., the velocity in the co-moving frame) that is important

- It is possible to do the transport in the lab frame

- Use cross sections for the neutron velocity in the co-moving frame

- The 'effective' total \& fission cross sections are not isotropic $\rightarrow$ complicates the algorithm \& may cause instabilities in the solution

- Alternatively, it is possible to transform the transport equation itself into the co-moving frame in which the material is stationary $\rightarrow$ "Moving Material Corrections" 


\section{Transport in Moving Materials}

- Transforming to the co-moving frame introduces spatial and momentum advection terms $\frac{\partial N}{\partial t}+\vec{q} \cdot \vec{\nabla}_{r} N+\vec{\nabla}_{r} \cdot \vec{u} N+\Sigma_{t} q N(\vec{r}, \vec{q}, t)=\dot{q}(\vec{r}, \vec{q}, t)+\vec{\nabla}_{q} \cdot \vec{H} N(\vec{r}, \vec{q}, t)$

$$
\begin{aligned}
& \vec{\nabla}_{q}=\frac{\vec{q}}{q}\left(\frac{\partial}{\partial q}-\frac{\mu}{q} \frac{\partial}{\partial \mu}\right)+\frac{\vec{r}}{r} \frac{1}{q} \frac{\partial}{\partial \mu} \\
& \vec{H}=\left(\vec{q} \cdot \vec{\nabla}_{r}+\vec{u} \cdot \vec{\nabla}_{r}+\frac{\partial}{\partial t}\right) \vec{u}
\end{aligned}
$$

- $\vec{q}=\vec{v}-\vec{u}$ is the neutron velocity in the co-moving frame (material at rest)

- $\vec{u}$ is the material velocity in the lab frame

- $N=\frac{\psi}{q}$ is the neutron density in the co-moving frame

- $\dot{q}$ is a source rate term (scattering, fission, volumetric sources)

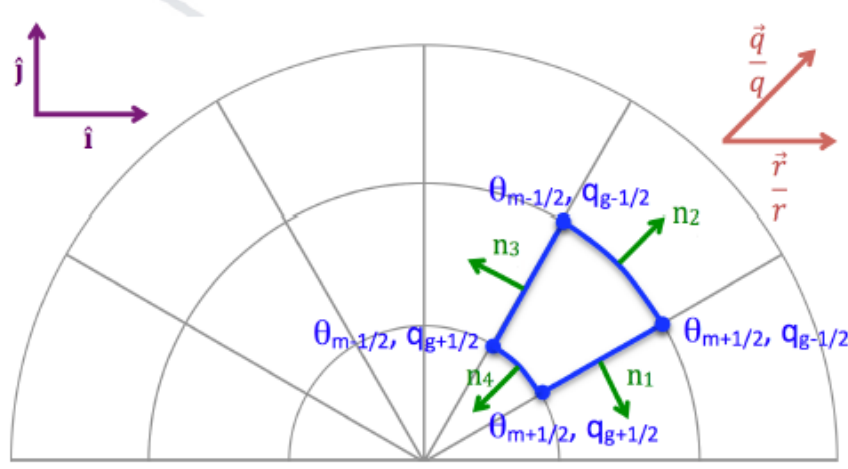

- Solve using a three-step operator split procedure:

1. Standard Transport step

2. Spatial Advection step

3. Momentum Advection step 


\section{Modeling R-Process Nucleosynthesis in Proto-Neutron Stars}

- Core-collapse supernovae are powered by the collapse of a stellar core into either a neutron star or a black hole

- Energy released powers an explosion that disrupts the star \& some material falls back onto the newly formed neutron star, creating secondary outbursts

- Fryer et al. argued that these outbursts could be the site of r-process nucleosynthesis, i.e., the formation of elements through the rapid capture of neutrons onto heavy seed nuclei

- This rapid neutron capture process is believed to be responsible for the creation of approximately one half of the atomic nuclei in the universe that are heavier than iron

- In this scenario, heavy 'seed' nuclei (e.g., iron) undergo a series of rapid neutron captures as they fall back toward the neutron star \& are then swept up in the outflow

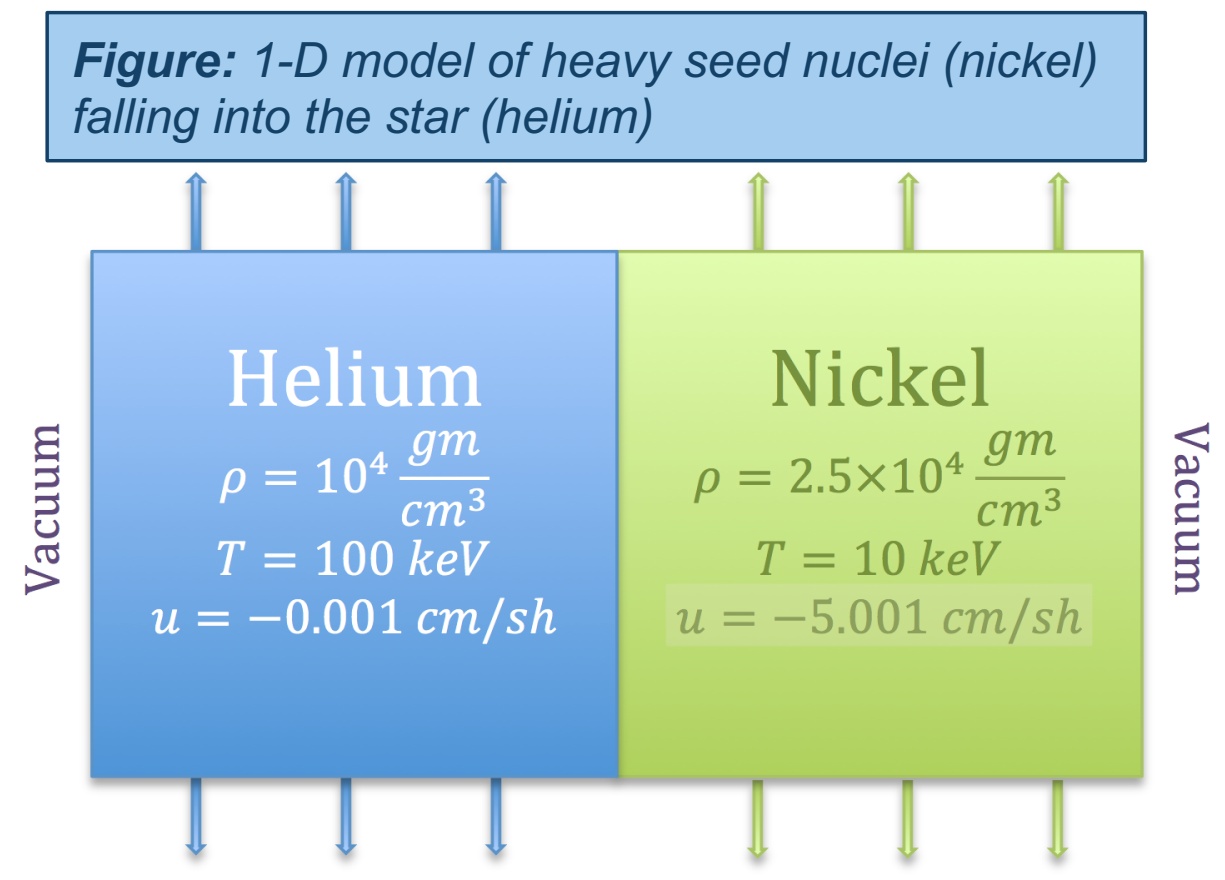




\section{Modeling R-Process Nucleosynthesis in Proto-Neutron Stars}

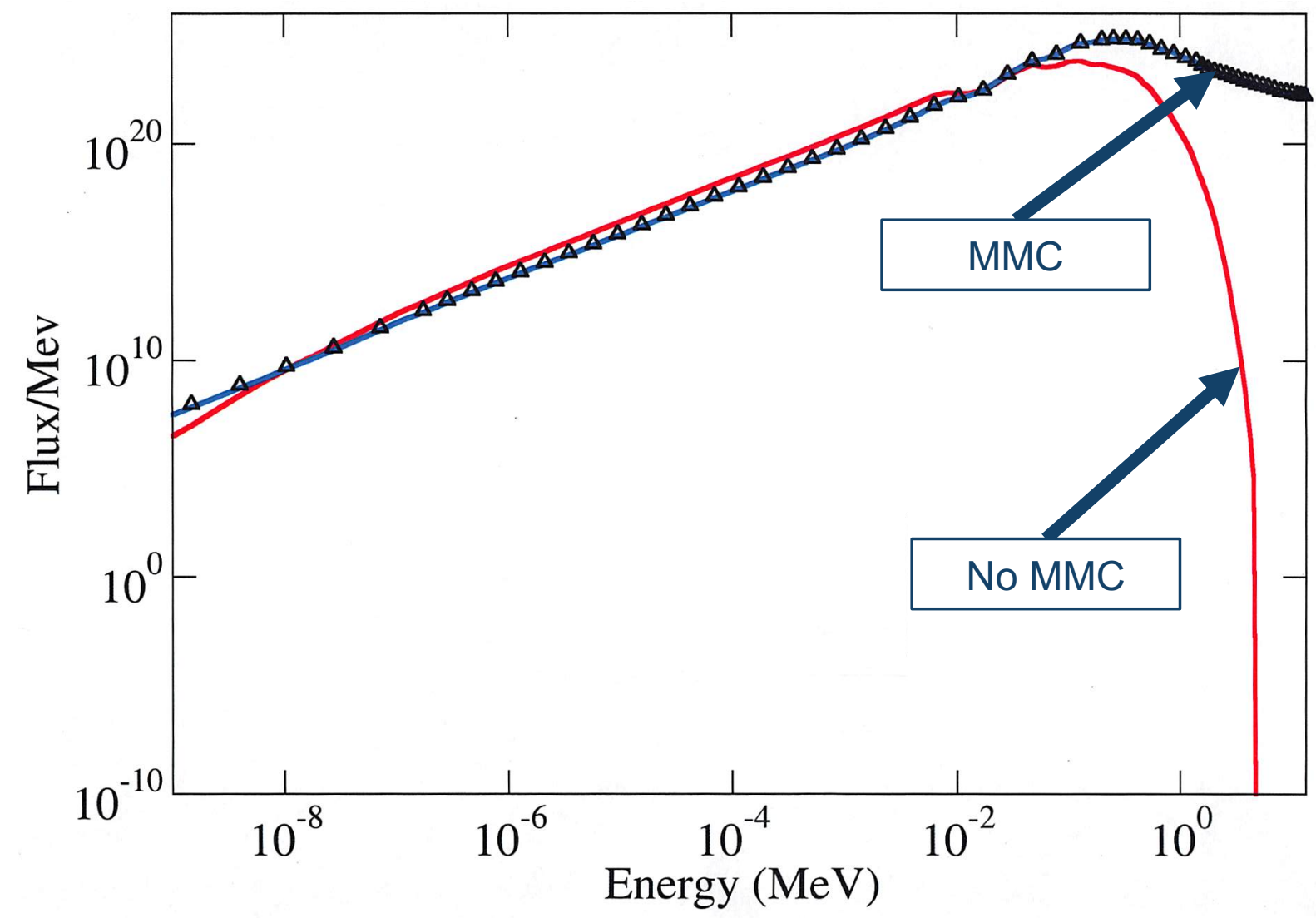

Figure: Neutron Spectrum at the $\mathrm{Ni} / \mathrm{He}$ interface Accounting for material motion changes the energy spectrum of the neutrons, which affects reaction rates 


\section{Sensitivity Analysis with SENSMG Jeffrey Favorite}




\section{SENSMG}

- SENSMG is a tool developed at LANL for calculating first-order sensitivities to multigroup cross sections, isotope densities, material mass densities, and interface locations using PARTISN and Generalized Perturbation Theory

- Reaction-rate ratios, $k_{e f f}$, and $\alpha$ in critical problems

- Reaction-rate ratios, reaction rates, and leakage in fixed-source problems

Jeffrey A. Favorite (2018) SENSMG: First-Order Sensitivities of Neutron Reaction Rates, Reaction-Rate Ratios, Leakage, $k_{e f f}$, and $\alpha$ Using PARTISN, Nuclear Science and Engineering, 192:1, 80-114, DOI: 10.1080/00295639.2018.1471296

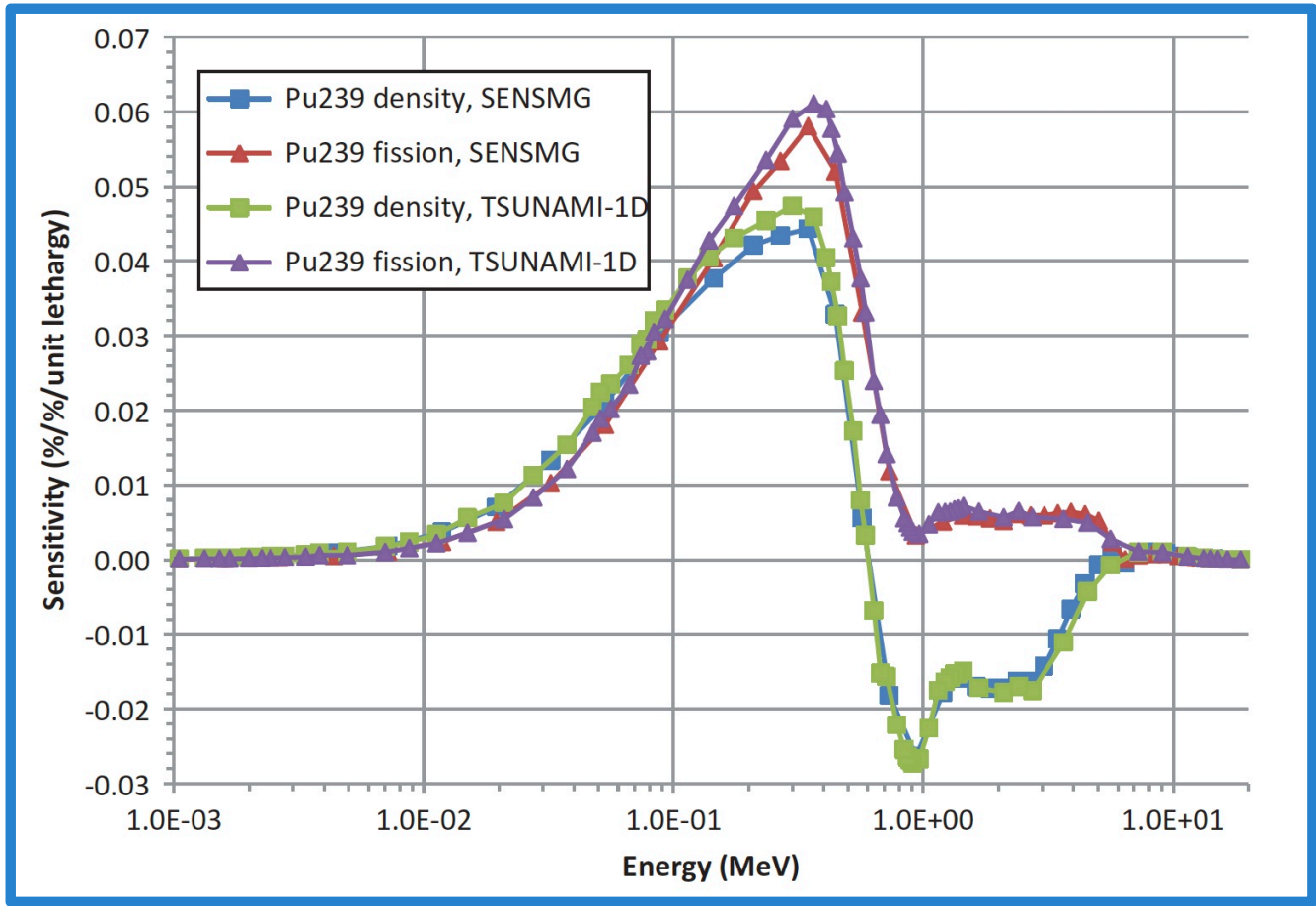

Figure: SENSMG vs. TSUNAMI-1D for Flattop-Pu Sensitivity of the ratio of ${ }^{237} \mathrm{~Np}$ fission to ${ }^{235} \mathrm{U}$ fission to the ${ }^{239} \mathrm{Pu}$ density and fission cross section as a function of energy 
Thank you for the opportunity to talk about PARTISN!

Questions? 